\title{
ON QUANTUM HYDRODYNAMIC AND QUANTUM ENERGY TRANSPORT MODELS*
}

\author{
PIERRE DEGOND ${ }^{\dagger}$, SAMY GALLEGO ${ }^{\ddagger}$, AND FLORIAN MÉHATS ${ }^{\S}$
}

\begin{abstract}
In this paper, we consider two recently derived models: the Quantum Hydrodynamic model (QHD) and the Quantum Energy Transport model (QET). We propose different equivalent formulations of these models and we use a commutator formula for stating new properties of the models. A gauge invariance lemma permits to simplify the QHD model for irrotational flows. We finish by considering the special case of a slowly varying temperature and we discuss possible approximations which will be helpful for future numerical discretizations.
\end{abstract}

Key words. density operator, quantum Liouville equation, quantum entropy, quantum local equilibrium, quantum hydrodynamics, quantum energy transport, commutators, gauge invariance

AMS subject classifications. 82C10, 82C70, 82D $37,81 \mathrm{Q} 05,81 \mathrm{~S} 05,81 \mathrm{~S} 30,81 \mathrm{~V} 70$

\section{Introduction}

This paper is the continuation of a series of works investigating the properties and numerical approximations of quantum hydrodynamics and diffusion models based on the entropy principle. The growing interest of the scientific community towards quantum macroscopic models arises from the fact that they are computationally less expensive than microscopic models such as the Schrödinger or Wigner equation [3, 4, $9,30,32,33]$. Simultaneously, collisions can be modeled without the use of quantum collision operators which are difficult to handle. The modeling of both quantum effects and collisions is particularly important for semiconductor devices where the active zone is small (sometimes less than 100 nanometers) and quantum effects are dominant, while the access zones are constituted of electron reservoirs in which collisions are predominant and drive the system towards thermodynamic equilibrium. An example of such a device is the resonant tunneling diode [7], which constitutes a good candidate for testing models, since it can be approximated by a one dimensional device.

The route which usually has been followed for the derivation of quantum hydrodynamics and diffusion models consists in incorporating some "quantum" correction terms, often based on the Bohm potential, into classical fluid models. This Bohm potential appears naturally in the fluid formulation of the Schrödinger equation for a single particle evolving in an external potential $V$. One obtains this formulation through the use of the Madelung Transform, which consists in writing the wave function in an exponential form $\psi=\sqrt{n} e^{i S / \hbar}$, where $n$ is the density of mass, $S$ is the phase and $\hbar$ denotes the scaled Planck constant. Inserting this ansatz into the Schrödinger equation, taking the real part and the gradient of the imaginary part, we recover the "Madelung equations," consisting in a pressureless Euler system involving an additional potential, called the Bohm potential $\frac{\hbar^{2}}{2} \Delta \sqrt{n} / n$. The first use of this Bohm potential in the semiconductor context dates back to $[1,2]$, in which the Bohm poten-

*Received: July 11, 2007; accepted (in revised version): September 6, 2007. Communicated by Shi Jin.

${ }^{\dagger}$ IMT, Université Paul Sabatier, 118 Route de Narbonne, 31062 Toulouse Cedex 4, France (degond @mip.ups-tlse.fr).

${ }^{\ddagger}$ IMT, Université Paul Sabatier, 118 Route de Narbonne, 31062 Toulouse Cedex 4, France (gallego @mip.ups-tlse.fr).

§IRMAR, Universit de Rennes, Campus de Beaulieu, 35042 Rennes Cedex, France (florian.mehats @univ-rennes1.fr). 
tial is added to the Drift-Diffusion equations and gives rise to the so-called DensityGradient model. This model has been used and studied in many articles [6, 28, 31]. In a diffusive setting, we can also cite [8] in which the Bohm potential is added to the Energy Transport model. In an hydrodynamic setting, many models including quantum corrections have been derived, and one can cite [19, 21, 22, 23, 24, 25, 26, 27].

New quantum hydrodynamic-like models have been constructed in [15] by applying the moment method to the quantum framework. These macroscopic models differ from the previous ones by the fact that they are fully quantum and do not rely on a perturbative approach when the scaled Planck constant is small. The derivation method consists in integrating the quantum Liouville equation with respect to the momentum $p$ against a given vector of polynomials of $p$. Following the approach that Levermore [29] developed in the classical case, the moment system is closed by a quantum local equilibrium defined as the solution of the minimization problem for the quantum entropy subject to the constraints that its moments are given. The quantum entropy being defined globally as the trace of an operator, the relation between the extensive variables (the chosen moments) and the thermodynamic intensive variables (the Lagrange multipliers of the constraints) is non-local. An interesting special case of the quantum moment method is obtained by choosing the hydrodynamic moments $\left(1, p,|p|^{2} / 2\right)$ as the moment system. This leads to the Quantum Hydrodynamic (QHD) model describing the evolution of the mass density $n$, the current density $n u$ and the energy density $\mathcal{W}$. More recently, Diffusion models have been derived in [14], namely the Quantum Energy Transport (QET) and the Quantum Drift-Diffusion (QDD) model, through diffusion limits of a collisional Wigner equation. The BGKlike collision operator used in this derivation has been constructed in order to relax the Wigner distribution function towards a quantum local equilibrium. The QDD model has been further studied in [13], and a numerical scheme for solving it has been proposed in [18]. Numerical simulations of a resonant tunneling diode and comparisons with other existing models have been reported in [10]. These simulations showed that the QDD model can provide reliable simulations of diffusive transport in nanoscale devices. Then, even more recently, the isothermal version of the QHD model (called the isothermal Quantum Euler system) has been studied in [11]. Many interesting properties of this model such as gauge invariance have been proved, and preliminary numerical simulations have been shown. A review of all this work can be found in [12].

In this paper, we consider non isothermal models, such as the QHD and QET models, and investigate some important properties of these models. These properties are important to take care of in numerical simulations. Previous works about numerical approximations of the QDD and Quantum Euler models [18, 10, 11] have been based on the simplification of the models by means of exact commutator relations. This strategy allowed to obtain partial differential equations describing the evolutions of the moments in terms of the moments themselves and their dual variables (i.e., the Lagrange multipliers of the constraints in the minimization problem). The quantum feature appears in the constitutive equations which give the link between the moments and their dual Lagrange multipliers. This constitutive relation supposes the diagonalization of a suitably defined Hamiltonian (see Section 2). It appears that this becomes more complex when an energy equation is added, and such a simplification is not possible in general. The evolution equations on the moments involve quantities that cannot be written in terms of a simple differential form of the moments and of their dual variables. Therefore numerical discretization will require to write 
the unknown moments in terms of the spectrum of the above mentioned Hamiltonian. Nevertheless, we can derive some differential constraints that link the moments and their dual Lagrange multipliers. These constraints need to be correctly approximated in any reliable numerical discretization. These constraints can be obtained via a lemma about commutators of operators the symbols of which are of the form $\lambda(x) p^{\alpha}$, where $\alpha$ is a multi-index $\alpha=\left(\alpha_{1}, \alpha_{2}, \alpha_{3}\right)$, i.e., $\lambda(x) p^{\alpha}=\lambda(x) p_{1}^{\alpha_{1}} p_{2}^{\alpha_{2}} p_{3}^{\alpha_{3}}$. This commutator relation was not written before (to the knowledge of the authors) and possesses an intrinsic value for future developments in this field.

The paper is organized as follows: In Section (2), we recall briefly the derivation of the QHD and QET models that can be found with more details in $[15,13,14]$. In particular, in Subsection (2.1) we recall the notion of quantum entropy and quantum local equilibrium, before stating the models themselves in Subsection (2.2) and (2.3).

In Section (3), we give four preliminary technical lemmas, the most important ones being Lemma (3.2), which allows us to write all the moments and their derivatives with respect to the discrete spectrum of the modified Hamiltonian, and Lemma (3.4), which gives the above-mentioned commutator relation. Note that these lemmas are useful for any quantum moment system derived by the method given in [15].

Then, Section (4) deals with the remarkable properties of the QHD model. Application of the preliminary technical lemmas first allows us to write all the quantities of the QHD model in terms of the spectrum of the modified Hamiltonian defined in Section (2) Lemma (4.1). It also allows us to state the differential constraints that link the moments and their dual Lagrange multipliers, Theorem (4.2). Again, these constraints should properly be taken into account in any reliable discretization of the system. Then, in Subsection (4.2), we state a gauge invariance Lemma (4.3) which leads to gauge transformations formulas (Lemma 4.4) and finally to a major simplification of the QHD model for irrotational flows (Theorem 4.5 and its Corollary 4.6). From these simplifications emerges a dispersive velocity term which has been found in other QHD derivations and which is discussed in Subsection (4.2.2). In Subsection (4.2.3) we discuss the special case of the QHD model in one dimension. In Subsection (4.3), we finally discuss some simplifications of the fluxes of the QHD model when the temperature is supposed to vary slowly.

Section (5) deals with the properties of the QET model. Like for the QHD model, application of preliminary technical lemmas first allows us to write the QET model in terms of the spectrum of the modified Hamiltonian defined in Section (2) Lemma (5.3). Application of the commutator Lemma (3.4) also allows us to derive the differential constraints between the moments and their Lagrange multipliers, Theorem (5.2), which any future discretization should take into account. In Subsection (5.2) possible simplifications for the fluxes of the QET model with slowly varying temperature are discussed.

Finally, in Section (6) we finish by giving a conclusion and some possible perspectives. We wish to specify that the arguments presented in this article are formal. A precise mathematical framework in which this analysis could be made rigorous is still an open subject.

\section{Context}

This section is a summary of previous work dealing with the Quantum Hydrodynamic model [15, 13] and the Quantum Energy Transport model [14]. This summary is given here to set up the context and the notation, and for the clarity of the presentation. 
2.1. Quantum entropy and quantum local equilibrium. By a density operator, we shall always mean a positive, Hermitian, trace-class operator acting on $L^{2}\left(\mathbb{R}^{3}\right)$. Let us define the first moments of a density operator $\varrho$, i.e., the mass density $n$, the current density $n u$ and the energy density $\mathcal{W}$, by duality, considering scalar test functions $\phi$ and vector test functions $\Phi$. We set

$$
\begin{aligned}
\forall \phi \in C_{0}^{\infty}\left(\mathbb{R}^{3}\right) \quad \int n \phi d x=\operatorname{Tr}(\varrho \phi), \\
\forall \Psi \in C_{0}^{\infty}\left(\mathbb{R}^{3}\right)^{3} \quad \int n u \cdot \Psi d x=\operatorname{Tr}\left(\varrho W^{-1}(\Psi \cdot p)\right) \\
=-i \hbar \operatorname{Tr}\left(\varrho\left(\Psi \cdot \nabla+\frac{1}{2}(\nabla \cdot \Psi)\right)\right), \\
\forall \phi \in C_{0}^{\infty}\left(\mathbb{R}^{3}\right) \quad \int \mathcal{W} \phi d x= \\
=\operatorname{Tr}\left(\varrho W^{-1}\left(\phi \frac{|p|^{2}}{2}\right)\right) \\
=-\frac{\hbar^{2}}{2} \operatorname{Tr}\left(\varrho\left(\nabla \cdot(\phi \nabla)+\frac{1}{4} \Delta \phi\right)\right) .
\end{aligned}
$$

In (2.2) and in (2.3), $W^{-1}$ denote the inverse Wigner transform (or Weyl quantization). For the sake of completeness, let us recall the definition of the Wigner transform and the inverse Wigner transform. The Wigner transform maps operators on $L^{2}\left(\mathbb{R}^{3}\right)$ to symbols, i.e., $L^{2}\left(\mathbb{R}^{3} \times \mathbb{R}^{3}\right)$ functions of the classical position and momentum variables $(x, p) \in \mathbb{R}^{3} \times \mathbb{R}^{3}$. More precisely, one defines the integral kernel of the operator $\varrho$ to be the distribution $\varrho\left(x, x^{\prime}\right)$ such that $\varrho$ operates on any function $\psi(x) \in L^{2}\left(\mathbb{R}^{3}\right)$ as follows:

$$
\varrho \psi(x)=\int \underline{\varrho}\left(x, x^{\prime}\right) \psi\left(x^{\prime}\right) d x^{\prime} .
$$

Then, the Wigner transform $W(\varrho)(x, p)$ is defined by:

$$
W(\varrho)(x, p)=\int \varrho\left(x-\frac{1}{2} \eta, x+\frac{1}{2} \eta\right) e^{\frac{i \eta \cdot p}{\hbar}} d \eta .
$$

The Wigner transform can be inverted and its inverse is defined for any function $w(x, p)$ as the operator acting on $\psi(x) \in L^{2}\left(\mathbb{R}^{3}\right)$ as:

$$
W^{-1}(w) \psi(x)=\int w\left(\frac{x+y}{2}, p\right) \psi(y) e^{\frac{i p \cdot(x-y)}{\hbar}} \frac{d p d y}{(2 \pi \hbar)^{3}} .
$$

The Wigner Transform $W$ and its inverse $W^{-1}$ are isometries between the space of Hilbert-Schmidt operators $\mathcal{L}^{2}$ (the space of operators such that the product $\varrho \varrho^{\dagger}$ is trace class, where $\varrho^{\dagger}$ is the Hermitian conjugate of $\varrho$ ) and the Hilbert space $L^{2}\left(\mathbb{R}^{3} \times \mathbb{R}^{3}\right)$ :

$$
\operatorname{Tr}\left(\varrho \sigma^{\dagger}\right)=\int W(\varrho)(x, p) \overline{W(\sigma)(x, p)} \frac{d x d p}{(2 \pi \hbar)^{3}} .
$$

This property allows us to define the mass density, the momentum density and the energy density in the Wigner picture, where we denote $W(\varrho)=w$ :

$$
\left(\begin{array}{c}
n \\
n u \\
\mathcal{W}
\end{array}\right)=\int w\left(\begin{array}{c}
1 \\
p \\
\frac{|p|^{2}}{2}
\end{array}\right) \frac{d p}{(2 \pi \hbar)^{3}}
$$


Definition 2.1. Let $s$ be a strictly convex continuously differentiable function on $\mathbb{R}^{+}$. We define the quantum entropy by:

$$
\mathscr{S}(\varrho)=\operatorname{Tr}(s(\varrho))
$$

Let the functions $n, n u$ and $\mathcal{W}$ be given and consider the following constrained minimization problem:

$\min \{\mathscr{S}(\varrho)$ such that $\varrho$ is a density operator satisfying (2.1), (2.2) and (2.3) $\}$.

The solution, if it exists, is called the local equilibrium density operator associated to $n$, nu and $\mathcal{W}$. Lagrange multiplier theory for the constrained problem (2.9) (see [15]) shows that there exist scalar functions $\tilde{A}$ and $\tilde{C}$, and a vector function $\tilde{B}$, all real valued and defined on $\mathbb{R}^{3}$, such that this local equilibrium density operator takes necessarily the form:

$$
\varrho_{n, n u, \mathcal{W}}^{e q}=\left(s^{\prime}\right)^{-1}(\tilde{H}(\tilde{A}, \tilde{B}, \tilde{C})),
$$

where $\tilde{H}(\tilde{A}, \tilde{B}, \tilde{C})$ is the following modified Hamiltonian:

$$
\begin{aligned}
\tilde{H}(\tilde{A}, \tilde{B}, \tilde{C}) & =W^{-1}\left(\tilde{A}+\tilde{B} \cdot p+\tilde{C}|p|^{2}\right) \\
& =\tilde{A}-i \hbar\left(\tilde{B} \cdot \nabla+\frac{1}{2}(\nabla \cdot \tilde{B})\right)-\hbar^{2}\left(\nabla \cdot(\tilde{C} \nabla)+\frac{1}{4} \Delta \tilde{C}\right) .
\end{aligned}
$$

It is convenient to change the entropic variables $(\tilde{A}, \tilde{B}, \tilde{C})$ into more physical variables $(A, B, C)$ in order to write the equilibrium density operator in the form:

$$
\varrho_{n, n u, \mathcal{W}}^{e q}=\left(s^{\prime}\right)^{-1}(-H(A, B, C)),
$$

where

$$
\begin{aligned}
H(A, B, C)= & W^{-1}\left(\frac{1}{2 C}(p-B)^{2}+A\right) \\
= & -\hbar^{2}\left(\nabla \cdot\left(\frac{1}{2 C} \nabla\right)+\frac{1}{4} \Delta \frac{1}{2 C}\right) \\
& +i \hbar\left(\frac{B}{C} \cdot \nabla+\frac{1}{2}\left(\nabla \cdot \frac{B}{C}\right)\right)+A+\frac{B^{2}}{2 C} .
\end{aligned}
$$

We will call the variables $A, B$ and $C$, respectively, the generalized chemical potential, the generalized mean velocity and the generalized temperature.

The link between $(\tilde{A}, \tilde{B}, \tilde{C})$ and $(A, B, C)$ is given by:

$$
A=-\tilde{A}+\frac{\tilde{B}^{2}}{4 \tilde{C}} ; B=-\frac{\tilde{B}}{2 \tilde{C}} ; C=-\frac{1}{2 \tilde{C}}
$$

or equivalently by:

$$
\tilde{A}=-A-\frac{B^{2}}{2 C} ; \tilde{B}=\frac{B}{C} ; \tilde{C}=-\frac{1}{2 C}
$$

This definition is obviously incomplete if no assumption is made on $n, n u$ and $\mathcal{W}$. In fact, this result has to be understood only at a formal level. Several crucial 
questions remain open: in which functional spaces $n, n u$ and $\mathcal{W}$ have to be chosen, in which spaces $A, B$ and $C$ have to be sought, and the question of existence and uniqueness of $A, B$ and $C$. Throughout this paper, we shall postpone this delicate question of realizability of moments, assuming that, as soon as the minimization problem (2.9) has to be solved, $n, n u$ and $\mathcal{W}$ are such that the associate functions $A$, $B$ and $C$ are uniquely defined. Note that even in the classical setting, the question of the realizability of moments for the constrained extremal problem is already a delicate question as pointed out in [16].

2.2. The Quantum Hydrodynamic model (QHD). In order to derive the QHD model, we start from the collisional quantum Liouville equation:

$$
i \hbar \partial_{t} \varrho=[\mathcal{H}, \varrho]+i \hbar \frac{\mathcal{Q}(\varrho)}{\varepsilon},
$$

where $\mathcal{H}$ is the Hamiltonian:

$$
\mathcal{H}=-\frac{\hbar^{2}}{2} \Delta+V
$$

and $\mathcal{Q}(\varrho)$ is an unspecified collision operator which describes the interaction of the particles with themselves and with their environment and accounts for dissipation mechanisms ( $\varepsilon$ is the scaled relaxation time). The key property that we request is that it drives the system to the local equilibria defined in the previous subsection. This is a consequence of the two following assumptions:

(i) mass, current and energy are conserved during collision, i.e., for any density operator $\varrho$ we have

$$
\forall \varphi, \Psi \quad \operatorname{Tr}\left(\mathcal{Q}(\varrho) W^{-1}\left(\begin{array}{c}
\varphi \\
\Psi \cdot p \\
\varphi \frac{|p|^{2}}{2}
\end{array}\right)\right)=0, \text { and }
$$

(ii) the quantum entropy is dissipated, except for the density operator in the kernel of $\mathcal{Q}$, which is explicitely described as follows:

$$
\mathcal{Q}(\varrho)=0 \text { iff } \exists(A, B, C) \text { such that } \varrho=\left(s^{\prime}\right)^{-1}(-H(A, B, C)) .
$$

Taking the Wigner Transform of (2.16), we get the following collisional Wigner equation for $w=W(\varrho)$ :

$$
\partial_{t} w+p \cdot \nabla w-\Theta^{\hbar}(V) w=\frac{Q(w)}{\varepsilon},
$$

with

$$
\Theta^{\hbar}(V) w=\frac{i}{(2 \pi)^{3} \hbar} \int\left(V\left(x+\frac{\hbar}{2} \eta\right)-V\left(x-\frac{\hbar}{2} \eta\right)\right) w(x, q) e^{i \eta \cdot(p-q)} d q d \eta,
$$

and $Q(w)$ is the Wigner Transform of $\mathcal{Q}(\varrho)$.

The moment method consists in taking the moments of the Wigner equation, i.e., multiplying it by $\left(\begin{array}{c}1 \\ p \\ \frac{|p|^{2}}{2}\end{array}\right)$ and integrating over $p$. Due to the conservation of mass, 
current and energy of the collision operator, and due to the properties of $\Theta^{\hbar}(V)$, we obtain the following system:

$$
\begin{aligned}
\partial_{t} n+\nabla \cdot n u & =0, \\
\partial_{t}(n u)+\nabla \cdot \Pi & =-n \nabla V, \\
\partial_{t} \mathcal{W}+\nabla \cdot \Phi & =-n u \cdot \nabla V,
\end{aligned}
$$

with the pressure tensor $\Pi$ and the energy flux $\Phi$ given by:

$$
\begin{aligned}
& \Pi=\int w(p \otimes p) \frac{d p}{(2 \pi \hbar)^{3}}, \\
& \Phi=\int w \frac{|p|^{2}}{2} p \frac{d p}{(2 \pi \hbar)^{3}} .
\end{aligned}
$$

It is readily seen that, with no further assumption, $\Pi$ and $\Phi$ cannot be expressed in terms of $n, n u$ and $\mathcal{W}$, meaning this system is not closed. Hence, by analogy with Levermore's methodology [29] and according to [15], we modify this system by replacing $w=W(\varrho)$ by the ansatz

$$
w_{n, n u, \mathcal{W}}^{e q}=W\left(\varrho_{n, n u, \mathcal{W}}^{e q}\right)=W\left(\left(s^{\prime}\right)^{-1}(-H(A, B, C))\right),
$$

which plays the role of a Maxwellian here. This ansatz corresponds to the modeling assumptions (i) and (ii) made on $\mathcal{Q}$, and represents the most likely quantum microscopic state which possesses the moments $n, n u$ and $\mathcal{W}$, according to the statistics that has been chosen, i.e., the function $s$ (this ansatz can also be justified by the hydrodynamic limit obtained when the scaled relaxation time $\varepsilon$ tends to 0 ). We obtain the QHD model, which consists of the mass, current and energy conservation equations (2.22-2.24), with:

$$
\left(\begin{array}{c}
n \\
n u \\
\mathcal{W}
\end{array}\right)=\int w_{n, n u, \mathcal{W}}^{e q}\left(\begin{array}{c}
1 \\
p \\
\frac{|p|^{2}}{2}
\end{array}\right) \frac{d p}{(2 \pi \hbar)^{3}}
$$

and

$$
\begin{aligned}
& \Pi=\int w_{n, n u, \mathcal{W}}^{e q}(p \otimes p) \frac{d p}{(2 \pi \hbar)^{3}}, \\
& \Phi=\int w_{n, n u, \mathcal{W}}^{e q} \frac{|p|^{2}}{2} p \frac{d p}{(2 \pi \hbar)^{3}} .
\end{aligned}
$$

2.3. The Quantum Energy Transport model (QET). We notice that the derivation of the QHD model did not require any knowledge of the exact form of the collision operator. For deriving a diffusion model such as the QET model from a kinetic equation, the exact form of the collision operator matters and the coefficients of the diffusion model itself depend on this collision operator. The most simple choice is a relaxation operator also called the BGK operator (for Bhatnagar, Gross, Krook [5]). The collision operator expresses the relaxation of the collision operator to the local thermodynamical equilibrium. We will choose:

$$
\mathcal{Q}(\varrho)=\varrho_{n, \mathcal{W}}^{e q}-\varrho,
$$


or in the Wigner picture:

$$
Q(w)=w_{n, \mathcal{W}}^{e q}-w
$$

where we have

$$
w_{n, \mathcal{W}}^{e q}=W\left(\varrho_{n, \mathcal{W}}^{e q}\right)=W\left(\left(s^{\prime}\right)^{-1}(-H(A, 0, C))\right),
$$

and the functions $A$ and $C$ are such that the operator $\mathcal{Q}$ conserves the mass and energy, i.e.,:

$$
\int W\left(\left(s^{\prime}\right)^{-1}(-H(A, 0, C))\right)\left(\begin{array}{c}
1 \\
\frac{|p|^{2}}{2}
\end{array}\right) \frac{d p}{(2 \pi \hbar)^{3}}=\left(\begin{array}{c}
n \\
\mathcal{W}
\end{array}\right) .
$$

We recall that the quantum equilibrium $\varrho_{n, \mathcal{W}}^{e q}$ is a solution of the entropy minimization principle: for $n$ and $\mathcal{W}$ given, to find

$$
\min \{\mathscr{S}(\varrho) \text { such that } \varrho \text { is a density operator satisfying }(2.1 \text { and } 2.3)\} \text {. }
$$

Now we consider a diffusion scaling of the collisional Wigner equation:

$$
\varepsilon^{2} \partial_{t} w^{\varepsilon}+\varepsilon\left(p \nabla w^{\varepsilon}-\Theta^{\hbar}(V) w^{\varepsilon}\right)=Q\left(w^{\varepsilon}\right),
$$

where the pseudo-differential operator $\Theta^{\hbar}(V)$ is defined by (2.21). This scaling is obtained through the change $t \rightarrow t / \varepsilon$, which means that we are looking at long time scales. The limit $\varepsilon \rightarrow 0$ of (2.36) is the QET model which consists of the following mass and energy conservation equations:

$$
\begin{array}{r}
\partial_{t} n+\nabla \cdot j_{n}=0, \\
\partial_{t} \mathcal{W}+\nabla \cdot j_{\mathcal{W}}+\nabla V \cdot j_{n}=0,
\end{array}
$$

where

$$
\begin{aligned}
j_{n} & =-\nabla \cdot \Pi-n \nabla V, \\
j_{\mathcal{W}} & =-\nabla \cdot \mathbb{Q}-(\mathcal{W} \operatorname{Id}+\Pi) \cdot \nabla V+\frac{\hbar^{2}}{8} n \nabla(\Delta V),
\end{aligned}
$$

with:

$$
\left(\begin{array}{c}
n \\
\mathcal{W}
\end{array}\right)=\int w_{n, \mathcal{W}}^{e q}\left(\begin{array}{c}
1 \\
\frac{|p|^{2}}{2}
\end{array}\right) \frac{d p}{(2 \pi \hbar)^{3}}
$$

and

$$
\begin{aligned}
& \Pi=\int w_{n, \mathcal{W}}^{e q}(p \otimes p) \frac{d p}{(2 \pi \hbar)^{3}}, \\
& \mathbb{Q}=\int w_{n, \mathcal{W}}^{e q}(p \otimes p) \frac{|p|^{2}}{2} \frac{d p}{(2 \pi \hbar)^{3}} .
\end{aligned}
$$




\section{Preliminary technical lemmas}

In this section, we give preliminary technical lemmas which are going to be useful for deriving properties of the QHD and QET models. Note that these lemmas can also be useful for any quantum moment systems that can be derived by applying the method explained in [15]. We start by giving a lemma that can be found in [15] (Lemma 3.1), and which proof is just an exercise in Fourier transform using Definition $(2.5)$ :

Lemma 3.1. Let $\alpha=\left(\alpha_{1}, \alpha_{2}, \alpha_{3}\right) \in \mathbb{N}^{3}$ be a multi-index (with $\mathbb{N}$ the set of natural integers) and write $p^{\alpha}=p_{1}^{\alpha_{1}} p_{2}^{\alpha_{2}} p_{3}^{\alpha_{3}}$ and $\partial_{x}^{\alpha}=\partial / \partial x_{1}^{\alpha_{1}} \partial / \partial x_{2}^{\alpha_{2}} \partial / \partial x_{3}^{\alpha_{3}}$. Then for any smooth real or complex valued function $\lambda(x)$, we have the following equivalent expression of the operator $W^{-1}\left(\lambda p^{\alpha}\right)$ :

$$
W^{-1}\left(\lambda p^{\alpha}\right)=(-i \hbar)^{|\alpha|} \sum_{0 \leq \gamma \leq \alpha}\left(\begin{array}{l}
\alpha \\
\gamma
\end{array}\right) \frac{1}{2^{|\gamma|}}\left(\partial_{x}^{\gamma} \lambda\right) \partial_{x}^{\alpha-\gamma},
$$

where $|\alpha|=\alpha_{1}+\alpha_{2}+\alpha_{3},\left(\begin{array}{l}\alpha \\ \gamma\end{array}\right)=\left(\begin{array}{c}\alpha_{1} \\ \gamma_{1}\end{array}\right)\left(\begin{array}{c}\alpha_{2} \\ \gamma_{2}\end{array}\right)\left(\begin{array}{c}\alpha_{3} \\ \gamma_{3}\end{array}\right)$ are the binomial coefficients and we denote $\sum_{0 \leq \gamma \leq \alpha}=\sum_{\gamma_{1}=0}^{\alpha_{1}} \sum_{\gamma_{2}=0}^{\alpha_{2}} \sum_{\gamma_{3}=0}^{\alpha_{3}}$.

As a consequence, we give the following lemma, which allows us to write the moments and the derivative of the moments associated to a quantum equilibrium $\varrho^{e q}=\left(s^{\prime}\right)^{-1}(-H)$, where $s$ is a convex function and $H$ an operator with a discrete spectrum (we use the same notations as in the previous lemma):

Lemma 3.2. Let $\alpha=\left(\alpha_{1}, \alpha_{2}, \alpha_{3}\right) \in \mathbb{N}^{3}$ and $\eta=\left(\eta_{1}, \eta_{2}, \eta_{3}\right) \in \mathbb{N}^{3}$ be two multi-indices. Suppose we have defined a quantum equilibrium $\varrho^{e q}=\left(s^{\prime}\right)^{-1}(-H)$, where $s$ is a convex function and $H$ an operator with a discrete spectrum that we denote by $\left(\lambda_{p}, \psi_{p}\right)_{p \in \mathbb{N}}$; then we have (see Lemma (3.1) for the notations):

$$
\begin{aligned}
& \partial_{x}^{\eta} \int p^{\alpha} W\left(\varrho^{e q}\right) \frac{d p}{(2 \pi \hbar)^{3}}=(-i \hbar)^{|\alpha|} \sum_{p \in \mathbb{N}}\left(s^{\prime}\right)^{-1}\left(-\lambda_{p}\right) \times \\
& \sum_{0 \leq \gamma \leq \alpha}\left(\begin{array}{c}
\alpha \\
\gamma
\end{array}\right)\left(-\frac{1}{2}\right)^{|\gamma|} \sum_{0 \leq \xi \leq \gamma+\eta}\left(\begin{array}{c}
\gamma+\eta \\
\xi
\end{array}\right)\left(\partial_{x}^{\alpha-\gamma+\xi} \psi_{p}\right)\left(\partial_{x}^{\gamma+\eta-\xi} \overline{\psi_{p}}\right) .
\end{aligned}
$$

Proof. Let $\phi(x)$ be a smooth test function; we have:

$$
\begin{aligned}
\int\left(\partial_{x}^{\eta} \int p^{\alpha} W\left(\left(s^{\prime}\right)^{-1}(-H)\right) \frac{d p}{(2 \pi \hbar)^{3}}\right) \phi d x \\
=(-1)^{|\eta|} \int p^{\alpha} W\left(\left(s^{\prime}\right)^{-1}(-H)\right) \partial_{x}^{\eta} \phi \frac{d p}{(2 \pi \hbar)^{3}} d x \\
=(-1)^{|\eta|} \operatorname{Tr}\left(W^{-1}\left(p^{\alpha} \partial_{x}^{\eta} \phi\right)\left(s^{\prime}\right)^{-1}(-H)\right) \\
=(-1)^{|\eta|} \sum_{p \in \mathbb{N}}\left(W^{-1}\left(p^{\alpha} \partial_{x}^{\eta} \phi\right)\left(s^{\prime}\right)^{-1}(-H) \psi_{p}, \psi_{p}\right)_{L^{2}\left(\mathbb{R}^{3}\right)} \\
=(-1)^{|\eta|} \sum_{p \in \mathbb{N}}\left(s^{\prime}\right)^{-1}\left(-\lambda_{p}\right)\left(W^{-1}\left(p^{\alpha} \partial_{x}^{\eta} \phi\right) \psi_{p}, \psi_{p}\right)_{L^{2}\left(\mathbb{R}^{3}\right)} .
\end{aligned}
$$




\section{QUANTUM HYDRODYNAMIC AND QUANTUM ENERGY TRANSPORT MODELS}

Then we use Lemma (3.1) to compute $W^{-1}\left(p^{\alpha} \partial_{x}^{\eta} \phi\right) \psi_{p}$, and the desired weak formulation is obtained after integrations by parts.

We recall now a lemma which can be found in [14] (Lemma 5.4) and whose proof is a direct application of pseudo-differential calculus (see for example [34]).

LEMMA 3.3. Let us consider two symbols $w_{1}(x, p)$ and $w_{2}(x, p)$ that are infinitely differentiable. The operation $w_{1} \circ_{\hbar} w_{2}$ gives the symbol of their operator product (this operation is sometimes noted $w_{1} \sharp w_{2}$ in the literature), i.e.,:

$$
w_{1} \circ_{\hbar} w_{2}=W\left(W^{-1}\left(w_{1}\right) W^{-1}\left(w_{2}\right)\right) .
$$

The following formal expansion holds:

$$
w_{1} \circ_{\hbar} w_{2}=\sum_{n=0}^{\infty} \hbar^{n} w_{1} \circ_{n} w_{2}
$$

with

$$
w_{1} \circ_{n} w_{2}(x, p)=\left(\frac{i}{2}\right)^{n} \sum_{\gamma, \zeta,|\gamma+\zeta|=n} \frac{(-1)^{|\gamma|}}{\gamma ! \zeta !} \partial_{x}^{\zeta} \partial_{p}^{\gamma} w_{1}(x, p) \partial_{x}^{\gamma} \partial_{p}^{\zeta} w_{2}(x, p),
$$

where we denote by $\lambda=\left(\lambda_{1}, \lambda_{2}, \lambda_{3}\right)$ and $\zeta=\left(\zeta_{1}, \zeta_{2}, \zeta_{3}\right)$ two multi-indices, and $\lambda !=$ $\lambda_{1} ! \lambda_{2} ! \lambda_{3}$ ! (see Lemma (3.1) for the other notations).

As a consequence, we give now a lemma which is helpful for commutator computations.

Lemma 3.4. Let $\alpha=\left(\alpha_{1}, \alpha_{2}, \alpha_{3}\right) \in \mathbb{N}^{3}$ and $\beta=\left(\beta_{1}, \beta_{2}, \beta_{3}\right) \in \mathbb{N}^{3}$ be two multi-indices, and let $\lambda(x)$ and $\mu(x)$ be any smooth real-or complex-valued functions. Let us denote by $\left[\lambda p^{\alpha}, \mu p^{\beta}\right]_{\hbar}$ the symbol associated to the commutator of the operators $W^{-1}\left(\lambda p^{\alpha}\right)$ and $W^{-1}\left(\mu p^{\beta}\right)$, i.e., :

$$
\left[\lambda p^{\alpha}, \mu p^{\beta}\right]_{\hbar}=W\left(\left[W^{-1}\left(\lambda p^{\alpha}\right), W^{-1}\left(\mu p^{\beta}\right)\right]\right) .
$$

The following formal expansion holds:

$$
\left[\lambda p^{\alpha}, \mu p^{\beta}\right]_{\hbar}=\sum_{k=0}^{\lfloor(|\alpha+\beta|-1) / 2\rfloor} \hbar^{2 k+1}\left[\lambda p^{\alpha}, \mu p^{\beta}\right]_{2 k+1},
$$

with

$$
\left[\lambda p^{\alpha}, \mu p^{\beta}\right]_{2 k+1}=i\left(-\frac{1}{4}\right)^{k} \sum_{\substack{0 \leq \gamma \leq \alpha, 0 \leq \zeta \leq \beta \\
|\gamma+\zeta|=2 k+1}}(-1)^{|\gamma|}\left(\begin{array}{c}
\alpha \\
\gamma
\end{array}\right)\left(\begin{array}{l}
\beta \\
\zeta
\end{array}\right)\left(\partial_{x}^{\zeta} \lambda\right)\left(\partial_{x}^{\gamma} \mu\right) p^{\alpha+\beta-\gamma-\zeta},
$$

where $\lfloor\cdot\rfloor$ denotes the floor function (see Lemma (3.1) for the other notations).

Proof. By the definition of the commutator and using notations of Lemma (3.3), we have:

$$
\begin{aligned}
{\left[\lambda p^{\alpha}, \mu p^{\beta}\right]_{\hbar} } & =\lambda p^{\alpha} \circ_{\hbar} \mu p^{\beta}-\mu p^{\beta} \circ_{\hbar} \lambda p^{\alpha} \\
& =\sum_{n=0}^{\infty} \hbar^{n}\left(\lambda p^{\alpha} \circ_{n} \mu p^{\beta}-\mu p^{\beta} \circ_{n} \lambda p^{\alpha}\right) .
\end{aligned}
$$


The operation $\circ_{n}$ being commutative (resp. anticommutative) when $n$ is even (resp. odd), we obtain:

$$
\left[\lambda p^{\alpha}, \mu p^{\beta}\right]_{\hbar}=\sum_{k=0}^{\infty} \hbar^{2 k+1}\left(2 \lambda p^{\alpha} \circ_{2 k+1} \mu p^{\beta}\right) .
$$

Then we apply the definition of the operation $\circ_{2 k+1}$ (3.6) to obtain the coefficients $\left[\lambda p^{\alpha}, \mu p^{\beta}\right]_{2 k+1}$, and we notice that these coefficients are zero as soon as $k>\lfloor(|\alpha+\beta|-$ $1) / 2\rfloor$.

\section{Remarkable properties of QHD}

4.1. Applications of the technical lemmas to QHD. All the quantities expressed in the QHD model can be written with respect to the spectrum of the modified Hamiltonian $H(A, B, C)$. This is what we do in the next lemma using Lemma (3.2).

Lemma 4.1. Suppose $H(A, B, C)$ has a discrete spectrum that we denote $\left(\lambda_{p}, \psi_{p}\right)_{p \in \mathbb{N}}$; then we have:

$$
\begin{aligned}
n & =\sum_{p \in \mathbb{N}}\left(s^{\prime}\right)^{-1}\left(-\lambda_{p}\right)\left|\psi_{p}\right|^{2}, \\
n u & =\hbar \sum_{p \in \mathbb{N}}\left(s^{\prime}\right)^{-1}\left(-\lambda_{p}\right) \operatorname{Im}\left(\nabla \psi_{p} \overline{\psi_{p}}\right) \\
\mathcal{W} & =\frac{\hbar^{2}}{4} \sum_{p \in \mathbb{N}}\left(s^{\prime}\right)^{-1}\left(-\lambda_{p}\right)\left(\left|\nabla \psi_{p}\right|^{2}-\mathcal{R e}\left(\Delta \psi_{p} \overline{\psi_{p}}\right)\right) .
\end{aligned}
$$

We can also express:

$$
\begin{aligned}
& \Pi=\frac{\hbar^{2}}{2} \sum_{p \in \mathbb{N}}\left(s^{\prime}\right)^{-1}\left(-\lambda_{p}\right) \operatorname{Re}\left(\nabla \psi_{p} \otimes \nabla \overline{\psi_{p}}-\overline{\psi_{p}} \nabla \otimes \nabla \psi_{p}\right), \\
& \Phi=-\frac{\hbar^{3}}{8} \sum_{p \in \mathbb{N}}\left(s^{\prime}\right)^{-1}\left(-\lambda_{p}\right) \operatorname{Im}\left(2\left(\nabla \otimes \nabla \overline{\psi_{p}}\right) \cdot \nabla \psi_{p}+\Delta \overline{\psi_{p}} \nabla \psi_{p}+\overline{\psi_{p}} \nabla \Delta \psi_{p}\right),
\end{aligned}
$$

and the fluxes:

$$
\begin{aligned}
& \nabla \cdot n u=\hbar \sum_{p \in \mathbb{N}}\left(s^{\prime}\right)^{-1}\left(-\lambda_{p}\right) \operatorname{Im}\left(\Delta \psi_{p} \overline{\psi_{p}}\right) \\
& \nabla \cdot \Pi=\frac{\hbar^{2}}{2} \sum_{p \in \mathbb{N}}\left(s^{\prime}\right)^{-1}\left(-\lambda_{p}\right) \operatorname{Re}\left(\nabla \psi_{p} \Delta \overline{\psi_{p}}-\overline{\psi_{p}} \nabla \Delta \psi_{p}\right), \\
& \nabla \cdot \Phi=-\frac{\hbar^{3}}{8} \sum_{p \in \mathbb{N}}\left(s^{\prime}\right)^{-1}\left(-\lambda_{p}\right) \operatorname{Im}\left(2 \nabla \psi_{p} \cdot \nabla \Delta \overline{\psi_{p}}+\overline{\psi_{p}} \Delta \Delta \psi_{p}\right) .
\end{aligned}
$$

Proof. Let $\left\{e_{1}, e_{2}, e_{3}\right\}$ denote the standard basis of $\mathbb{R}^{3}$. We use lem. (3.2) with:

- $\eta=(0,0,0)$ and $\alpha=(0,0,0)$ to get $(4.1)$,

- $\eta=(0,0,0)$ and $\alpha=e_{i}$ to get the $i^{\text {th }}$ component of (4.2),

- $\eta=(0,0,0)$ and $\alpha=2 e_{j}$ to get (4.3) after summation on $j$ and division by 2 , 
898 QUANTUM HYDRODYNAMIC AND QUANTUM ENERGY TRANSPORT MODELS

- $\eta=(0,0,0)$ and $\alpha=e_{i}+e_{j}$ to get the coefficient $(i, j)$ of (4.4),

- $\eta=(0,0,0)$ and $\alpha=e_{i}+2 e_{j}$ to get (4.5) after summation on $j$ and division by 2 ,

- $\eta=e_{j}$ and $\alpha=e_{j}$ to get (4.6) after summation on $j$,

- $\eta=e_{j}$ and $\alpha=e_{i}+e_{j}$ to get the $i^{\text {th }}$ component of (4.7) after summation on $j$.

- $\eta=e_{j}$ and $\alpha=2 e_{i}+e_{j}$ to get (4.8) after summation on $i$ and $j$ and division by 2 .

We now give an application of Lemma (3.4). The following theorem gives general relations involving $n, n u, \mathcal{W}, \Pi, \Phi$ and $A, B, C$ as soon as they are linked according to $(2.27-2.30)$. Any future discretization should be thought such that the following relations are respected.

Theorem 4.2. Let $n, n u, \mathcal{W}, \Pi, \Phi$ and $A, B, C$ satisfy (2.27-2.30). Then we have:

$$
\begin{aligned}
& \nabla \cdot \frac{n u}{C}=\nabla \cdot \frac{n B}{C} \\
& \nabla \cdot \frac{\Pi}{C}=\nabla \cdot\left(n u \otimes \frac{B}{C}\right)+\left(\nabla \frac{B}{C}\right) \cdot n u-n \nabla\left(A+\frac{B^{2}}{2 C}\right)-\mathcal{W} \nabla \frac{1}{C} \\
& +\frac{\hbar^{2}}{8} \Delta\left(n \nabla \frac{1}{C}\right) \\
& \nabla \cdot \frac{\Phi}{C^{2}}=\frac{1}{C} \Pi:\left(\nabla \frac{B}{C}\right)+\frac{1}{C} \nabla \cdot\left(\frac{B}{C} \mathcal{W}\right)-\frac{1}{C} \nabla\left(A+\frac{B^{2}}{2 C}\right) \cdot n u \\
& +\frac{\hbar^{2}}{8 C}\left(\nabla \cdot\left(n u \Delta \frac{1}{C}-n \Delta \frac{B}{C}\right)+\Delta\left(n u \cdot \nabla \frac{1}{C}\right)\right) \text {. }
\end{aligned}
$$

Proof. Let $\mu(x)$ be a smooth test function. Using Lemma (3.4), we compute:

$$
\begin{aligned}
& {[W(\tilde{H}(\tilde{A}, \tilde{B}, \tilde{C})), \mu]_{\hbar}=[\tilde{A}, \mu]_{\hbar}+[\tilde{B} \cdot p, \mu]_{\hbar}+\left[\tilde{C}|p|^{2}, \mu\right]_{\hbar}} \\
& =0-i \hbar(\tilde{B} \cdot \nabla \mu+2 \tilde{C} \nabla \mu \cdot p) \text {. } \\
& {[W(\tilde{H}(\tilde{A}, \tilde{B}, \tilde{C})), \mu p]_{\hbar}=[\tilde{A}, \mu p]_{\hbar}+[\tilde{B} \cdot p, \mu p]_{\hbar}+\left[\tilde{C}|p|^{2}, \mu p\right]_{\hbar}} \\
& =-i \hbar(-\mu \nabla \tilde{A}+(\tilde{B} \cdot \nabla \mu) p-\mu(\nabla \tilde{B}) \cdot p \\
& \left.+2 \tilde{C}(\nabla \mu \cdot p) p-\mu \nabla \tilde{C}|p|^{2}+\frac{\hbar^{2}}{4} \nabla \tilde{C} \Delta \mu\right) . \\
& {\left[W(\tilde{H}(\tilde{A}, \tilde{B}, \tilde{C})), \mu|p|^{2}\right]_{\hbar}=\left[\tilde{A}, \mu|p|^{2}\right]_{\hbar}+\left[\tilde{B} \cdot p, \mu|p|^{2}\right]_{\hbar}+\left[\tilde{C}|p|^{2}, \mu|p|^{2}\right]_{\hbar}} \\
& =-i \hbar\left(-2 \mu \nabla \tilde{A} \cdot p-2 \mu(p \otimes p):(\nabla \tilde{B})+\tilde{B} \cdot \nabla \mu|p|^{2}\right. \\
& -\frac{\hbar^{2}}{4} \Delta \tilde{B} \cdot \nabla \mu+2 \tilde{C} \nabla \mu \cdot p|p|^{2}-2 \mu \nabla \tilde{C} \cdot p|p|^{2} \\
& \left.-\frac{\hbar^{2}}{4}(2 \Delta \tilde{C} \nabla \mu \cdot p-2 \Delta \mu \nabla \tilde{C} \cdot p)\right) \text {. }
\end{aligned}
$$


Then due to the cyclicity of the trace, we write for the three commutators:

$$
\begin{array}{r}
\operatorname{Tr}\left(\left[\tilde{H}(\tilde{A}, \tilde{B}, \tilde{C}), W^{-1}\left(\mu p^{\alpha}\right)\right] \varrho_{n, n u, \mathcal{W}}^{e q}\right)=0 \\
=\int\left[W(\tilde{H}(\tilde{A}, \tilde{B}, \tilde{C})), \mu p^{\alpha}\right]_{\hbar} W\left(\varrho_{n, n u, \mathcal{W}}^{e q}\right) \frac{d x d p}{(2 \pi \hbar)^{3}}
\end{array}
$$

which gives weakly the three desired identities after integrations by parts and the change of variable given by (2.15).

\subsection{Gauge invariance and irrotational flows.}

4.2.1. Gauge invariance. We now turn to look at gauge invariance, an interesting property that will simplify the model for irrotational flows.

Lemma 4.3 (Gauge invariance). Let $\alpha=\left(\alpha_{1}, \alpha_{2}, \alpha_{3}\right) \in \mathbb{N}^{3}$ be a multi-index, and let $S(x)$ and $\lambda(x)$ be smooth functions. Then we have:

$$
e^{i S / \hbar} H(A, B, C) e^{-i S / \hbar}=H(A, B+\nabla S, C) .
$$

Proof. To prove identity (4.15), we remark that for $|\alpha| \leq 2$,

$$
e^{i S / \hbar} W^{-1}\left(\lambda p^{\alpha}\right) e^{-i S / \hbar}=W^{-1}\left(\lambda(p-\nabla S)^{\alpha}\right) .
$$

The modified Hamiltonian $H(A, B, C)=W^{-1}\left(\frac{1}{2 C}(p-B)^{2}+A\right)$ being an operator associated to a polynomial in $p$ of order 2 , we have:

$$
e^{i S / \hbar} W^{-1}\left(\frac{1}{2 C}(p-B)^{2}+A\right) e^{-i S / \hbar}=\left(\frac{1}{2 C}(p-(B+\nabla S))^{2}+A\right),
$$

which is (4.15).

This gauge invariance lemma allows us to write gauge transformations which are summarized in the next lemma.

Lemma 4.4. Let $n(A, B, C), n u(A, B, C), \mathcal{W}(A, B, C), \Pi(A, B, C)$ and $\Phi(A, B, C) d e-$ note the extensive quantities associated to the intensive quantities $(A, B, C)$ according to (2.27-2.30). Then we have the following identities:

$$
\begin{aligned}
n(A, B+\nabla S, C)= & n(A, B, C) \\
(n u)(A, B+\nabla S, C)= & (n u)(A, B, C)+n(A, B, C) \nabla S \\
\mathcal{W}(A, B+\nabla S, C)= & \mathcal{W}(A, B, C)+(n u)(A, B, C) \cdot \nabla S+\frac{1}{2} n(A, B, C)|\nabla S|^{2},( \\
\Pi(A, B+\nabla S, C)= & \Pi(A, B, C)+(n u)(A, B, C) \otimes \nabla S \\
& +\nabla S \otimes(n u)(A, B, C)+n(A, B, C) \nabla S \otimes \nabla S \\
\Phi(A, B+\nabla S, C)= & \Phi(A, B, C)+(\Pi(A, B, C)+\mathcal{W}(A, B, C) I d) \cdot \nabla S \\
& +\frac{1}{2} n(A, B, C)|\nabla S|^{2} \nabla S+\frac{1}{2}(n u)(A, B, C)|\nabla S|^{2} \\
& +((n u)(A, B, C) \cdot \nabla S) \nabla S-\frac{\hbar^{2}}{8} n(A, B, C) \nabla \Delta S
\end{aligned}
$$

which relate the mass, current, and energy densities, the pressure tensor and the energy flux for two values of $B$ differing by a gradient. 
Proof. Let $\alpha=\left(\alpha_{1}, \alpha_{2}, \alpha_{3}\right) \in \mathbb{N}^{3}$ be a multi-index and $\lambda$ be a smooth test function and let us denote $w^{e q}(A, B, C)=W\left(\left(s^{\prime}\right)^{-1}(-H(A, B, C))\right)$. Due to Lemma (4.3), we can write:

$$
\begin{aligned}
\int w^{e q}(A, B+\nabla S, C) \lambda p^{\alpha} \frac{d x d p}{(2 \pi \hbar)^{3}} & =\operatorname{Tr}\left(\left(s^{\prime}\right)^{-1}(-H(A, B+\nabla S, C)) W^{-1}\left(\lambda p^{\alpha}\right)\right) \\
& =\operatorname{Tr}\left(e^{i S / \hbar}\left(s^{\prime}\right)^{-1}(-H(A, B, C)) e^{-i S / \hbar} W^{-1}\left(\lambda p^{\alpha}\right)\right) \\
& =\operatorname{Tr}\left(\left(s^{\prime}\right)^{-1}(-H(A, B, C)) e^{-i S / \hbar} W^{-1}\left(\lambda p^{\alpha}\right) e^{i S / \hbar}\right) .
\end{aligned}
$$

Then, as we have already noticed earlier, we use the fact that for $|\alpha| \leq 2$,

$$
e^{-i S / \hbar} W^{-1}\left(\lambda p^{\alpha}\right) e^{i S / \hbar}=W^{-1}\left(\lambda(p+\nabla S)^{\alpha}\right),
$$

so that finally we have for $|\alpha| \leq 2$

$$
\int w^{e q}(A, B+\nabla S, C) \lambda p^{\alpha} \frac{d x d p}{(2 \pi \hbar)^{3}}=\int w^{e q}(A, B, C) \lambda(p+\nabla S)^{\alpha} \frac{d x d p}{(2 \pi \hbar)^{3}}
$$

which gives the following identity:

$$
\int w^{e q}(A, B+\nabla S, C) p^{\alpha} \frac{d p}{(2 \pi \hbar)^{3}}=\int w^{e q}(A, B, C)(p+\nabla S)^{\alpha} \frac{d p}{(2 \pi \hbar)^{3}} .
$$

Then we choose (denoting by $\left\{e_{1}, e_{2}, e_{3}\right\}$ the standard basis of $\mathbb{R}^{3}$ ):

- $\alpha=(0,0,0)$ to get $(4.16)$,

- $\alpha=e_{i}$ to get the $i^{\text {th }}$ component of (4.17),

- $\alpha=2 e_{j}$ to get (4.18) after summation on $j$ and division by 2 ,

- $\alpha=e_{i}+e_{j}$ to get the coefficient $(i, j)$ of (4.19).

In order to get (4.20), we compute for $\alpha=e_{i}+2 e_{j}$

$$
e^{-i S / \hbar} W^{-1}\left(\lambda p^{\alpha}\right) e^{i S / \hbar}=W^{-1}\left(\lambda(p+\nabla S)^{\alpha}\right)-\frac{\hbar^{2}}{4} \lambda \partial_{i} \partial_{j}^{2} S,
$$

and we get the following identity for $\alpha=e_{i}+2 e_{j}$ :

$$
\int w^{e q}(A, B+\nabla S, C) p^{\alpha} \frac{d p}{(2 \pi \hbar)^{3}}=\int w^{e q}(A, B, C)\left((p+\nabla S)^{\alpha}-\frac{\hbar^{2}}{4} \partial_{i} \partial_{j}^{2} S\right) \frac{d p}{(2 \pi \hbar)^{3}} .
$$

Finally we obtain the $i^{\text {th }}$ component of (4.20) after summation on $j$ and division by 2 .

4.2.2. Irrotational flows. It is now possible to give interesting properties for the special case of the QHD model with irrotational flows as stated in the next theorem.

Theorem 4.5. Let $n, n u, \mathcal{W}$ and $A, B, C$ be given according to Definition (2.1). Assume moreover that $u$ is an irrotational vector field, i.e., that there exists $S(x)$ such that $u=\nabla S$. Then $B$ is defined by

$$
B=u=\nabla S,
$$


and we have

$$
\varrho_{n, n u, \mathcal{W}}^{e q}=e^{i S / \hbar} \varrho_{n, 0, \mathcal{W}}^{e q} e^{-i S / \hbar},
$$

where the two equilibrium density operators $\varrho_{n, n u, \mathcal{W}}^{e q}$ and $\varrho_{n, 0, \mathcal{W}}^{e q}$ are given according to Definition (2.1). If we denote by $n(A, B, C)$, nu $(A, B, C), \mathcal{W}(A, B, C), \Pi(A, B, C)$ and $\Phi(A, B, C)$ the extensive quantities associated to the intensive quantities $(A, B, C)$ according to (2.27-2.30), we have moreover:

$$
\begin{aligned}
n(A, B, C)= & n(A, 0, C), \\
\mathcal{W}(A, B, C)= & \mathcal{W}(A, 0, C)+\frac{1}{2} n(A, 0, C)|u|^{2}, \\
\Pi(A, B, C)= & \Pi(A, 0, C)+n(A, 0, C) u \otimes u, \\
\Phi(A, B, C)= & (\Pi(A, 0, C)+\mathcal{W}(A, 0, C) I d) \cdot u+\frac{1}{2} n(A, 0, C)|u|^{2} u \\
& -\frac{\hbar^{2}}{8} n(A, 0, C) \Delta u .
\end{aligned}
$$

Proof. Let $n, n u$ and $\mathcal{W}$ be given and consider the following minimization problem with only two constraints: Find

$$
\begin{aligned}
& \min \{\mathscr{S}(\varrho) \text { such that the density associated to } \varrho \text { is } n, \\
& \text { see } \left.(2.1), \text { and the energy is } \mathcal{W}-\frac{1}{2} n u^{2}, \text { see }(2.3)\right\} .
\end{aligned}
$$

Following [14], this minimization problem (4.27) is attained on a density operator which reads

$$
\varrho_{0}=\left(s^{\prime}\right)^{-1}(-H(\alpha, 0, \gamma)),
$$

where $\alpha$ and $\gamma$ are two scalar functions and $H(\alpha, 0, \gamma)$ is still defined according to (2.11). Due to the fact that the Wigner function of $\varrho_{0}$ is even (see [14]), this density operator carries no current. This is enough to conclude that $\varrho_{0}=\varrho_{n, 0, \mathcal{W}-\frac{1}{2} n u^{2}}^{e q}$ (following Definition 2.1) or, equivalently, that

$$
n(\alpha, 0, \gamma)=n, \quad(n u)(\alpha, 0, \gamma)=0, \quad \mathcal{W}(\alpha, 0, \gamma)=\mathcal{W}-\frac{1}{2} n u^{2} .
$$

Denote now

$$
\varrho_{S}=\left(s^{\prime}\right)^{-1}(-H(\alpha, \nabla S, \gamma))
$$

Applying the Gauge invariance of Lemma (4.3), we have:

$$
H(\alpha, \nabla S, \gamma)=e^{i S / \hbar} H(\alpha, 0, \gamma) e^{-i S / \hbar} .
$$

It is immediate to deduce from elementary functional calculus that

$$
\varrho_{S}=e^{i S / \hbar} \varrho_{0} e^{-i S / \hbar} \text {. }
$$

Note then that, by definition, the mass density, the current density and the energy density corresponding to $\varrho_{S}$ are respectively $n(\alpha, \nabla S, \gamma),(n u)(\alpha, \nabla S, \gamma)$ and $\mathcal{W}(\alpha, \nabla S, \gamma)$, and, as a direct consequence of Lemma (4.4), we have

$$
\begin{aligned}
& n(\alpha, \nabla S, \gamma)=n(\alpha, 0, \gamma)=n, \\
& (n u)(\alpha, \nabla S, \gamma)=(n u)(\alpha, 0, \gamma)+n(\alpha, 0, \gamma) \nabla S=n u, \\
& \mathcal{W}(\alpha, \nabla S, \gamma)=\mathcal{W}(\alpha, 0, \gamma)+(n u)(\alpha, 0, \gamma) \nabla S+\frac{1}{2} n(\alpha, 0, \gamma)|\nabla S|^{2}=\mathcal{W} .
\end{aligned}
$$


Therefore, according to the property of uniqueness of the Lagrange multipliers $A, B$ and $C$ assumed in Subsection (2.1), we deduce that $A=\alpha, B=\nabla S$ and $C=\gamma$.

The last part of the theorem (identities 4.23-4.26) is a direct consequence of Lemma 4.4.

COROLLARY 4.6. For irrotational flows, the QHD model reads:

$$
\begin{aligned}
\partial_{t} n+\nabla \cdot n u & =0, \\
\partial_{t}(n u)+\nabla \cdot \Pi & =-n \nabla V, \\
\partial_{t} \mathcal{W}+\nabla \cdot\left((\Pi+\mathcal{W} I d) \cdot u-n|u|^{2} u-\frac{\hbar^{2}}{8} n \Delta u\right) & =-n u \cdot \nabla V,
\end{aligned}
$$

with

$$
\begin{aligned}
n & =\int w_{n, \mathcal{W}}^{e q} \frac{d p}{(2 \pi \hbar)^{3}} \\
\mathcal{W} & =\frac{1}{2} n|u|^{2}+\int w_{n, \mathcal{W}}^{e q} \frac{|p|^{2}}{2} \frac{d p}{(2 \pi \hbar)^{3}}
\end{aligned}
$$

and

$$
\Pi=n u \otimes u+\int w_{n, \mathcal{W}}^{e q}(p \otimes p) \frac{d p}{(2 \pi \hbar)^{3}}, .
$$

The quantum local equilibrium $w_{n, \mathcal{W}}^{e q}$ is defined by

$$
w_{n, \mathcal{W}}^{e q}=W\left(\varrho_{n, \mathcal{W}}^{e q}\right)=W\left(\left(s^{\prime}\right)^{-1}(-H(A, 0, C))\right),
$$

where $H(A, 0, C)$ is the following modified Hamiltonian:

$$
\begin{aligned}
H(A, 0, C) & =W^{-1}\left(\frac{1}{2 C} p^{2}+A\right) \\
& =-\hbar^{2}\left(\nabla \cdot\left(\frac{1}{2 C} \nabla\right)+\frac{1}{4} \Delta \frac{1}{2 C}\right)+A .
\end{aligned}
$$

Proof. The proof is straightforward using Theorem (4.5).

Let us summarize the simplifications obtained for the QHD model with irrotational flows. Firstly, two unknowns have been canceled, namely the energy flux $\Phi$ and the generalized mean velocity $B$. Secondly, the link between the moments and their dual variables is simpler. The underlying minimization problem is now (4.27), with only the constraint on the density and the energy, instead of (2.9). The local equilibrium $\varrho_{n, \mathcal{W}}^{e q}=\left(s^{\prime}\right)^{-1}(-H(A, 0, C))$ is the same as the one of the QET model, and in the case where its spectrum is discrete, this spectrum has the advantage of being real. In addition, notice that the dispersive velocity term $-\frac{\hbar^{2}}{8} \nabla \cdot(n \Delta u)$ appearing in the divergence of the energy flux (4.30) also appears in other QHD derivations. It has been derived in [20] from a mixed-state Wigner model and interpreted as a dispersive "heat flux". It also appears in the QHD equations of [22] involving a "smoothed" potential, derived from the quantum Liouville equation by a Chapman-Enskog expansion. Finally, it appears in [27] where an $\hbar$ expansion of the QHD model presented here (with a Boltzmann statistics) is performed. It has been noted in [27] that an interesting feature of this dispersive term is that it stabilizes the QHD system numerically. 
4.2.3. One-dimensional flows. A special case of irrotational flows is onedimensional flows. It is natural to wonder if the 1D QHD model can be written in a simple way. It appears that this is a delicate question and we see two possibilities:

1. If the transport is confined in one dimension (for example if we want to model quantum transport confined on a wire [17], we can suppose that the temperature is anisotropic and the momentum $p$ is confined on a line with coordinate $x_{1} \in \mathbb{R}$ ), then we can start from a $1 \mathrm{D}$ Wigner equation and define a 1D quantum local equilibrium, and in this case we obtain the following model:

$$
\begin{aligned}
\partial_{t} n+\partial_{x_{1}}(n u) & =0 \\
\partial_{t}(n u)+2 \partial_{x_{1}} \mathcal{W} & =-n \partial_{x_{1}} V, \\
\partial_{t} \mathcal{W}+\partial_{x_{1}}\left(3 \mathcal{W} u-n u^{3}-\frac{\hbar^{2}}{8} n \partial_{x_{1}}^{2} u\right) & =-n u \partial_{x_{1}} V .
\end{aligned}
$$

Notice that these 3 equations are now decoupled from the constitutive equations linking $(n, n u, \mathcal{W})$ to $(A, B, C)$. This model is exact (without any approximation) and its only quantum character reduces to the dispersive velocity term $-\frac{\hbar^{2}}{8} \partial_{x_{1}}\left(n \partial_{x_{1}}^{2} u\right)$. A surprising fact is that this model coincides with its $\hbar$ expansion up to second order, proving that the higher order terms are equal to zero independently of the chosen statistics. Notice also that in the semiclassical limit $(\hbar \rightarrow 0)$, we do not recover the classical hydrodynamic equations used in the literature.

2. If the problem is in dimension three (for the momentum $p$ ) but all the quantities depend only on one space variable (say on $x_{1}$ ), then the derivation of the $1 \mathrm{D}$ model from the 3D model (4.28-4.35) is not possible without some more assumptions. This statement needs to be made precise. Let us consider the stress tensor $\mathbb{P}=\int w_{n, \mathcal{W}}^{e q}(p \otimes p) \frac{d p}{(2 \pi \hbar)^{3}}$ appearing in the pressure tensor $\Pi$ (4.33). Without any assumption, this tensor is not diagonal, contrary to the classical case (as already noticed in [14]). With the assumption that all the quantities depend only on the variable $x_{1}$, and if we suppose that the function $\left(s^{\prime}\right)^{-1}(\cdot)$ is expandable as a power series, it is possible to show that $w_{n, \mathcal{W}}^{e q}$ is even with respect to $p_{2}$ and $p_{3}$. Using Lemma (3.3), we prove indeed that any power of $H(A, 0, C)$ is a symbol depending only on $\left(x_{1}, p\right)$ and even with respect to $p_{2}, p_{3}$, and thus $\mathbb{P}$ is diagonal. But nevertheless it is not scalar. If we suppose moreover that $\mathbb{P}$ is scalar, using the constraint that its trace must be equal to $2 \mathcal{W}-n|u|^{2}$, we can suppose that $\mathbb{P}=\left(2 \mathcal{W}-n|u|^{2}\right) / 3 \mathrm{Id}$. We obtain then the following 1D QHD model:

$$
\begin{aligned}
\partial_{t} n+\partial_{x_{1}}(n u) & =0 \\
\partial_{t}(n u)+\frac{2}{3} \partial_{x_{1}}\left(\mathcal{W}+n u^{2}\right) & =-n \partial_{x_{1}} V \\
\partial_{t} \mathcal{W}+\partial_{x_{1}}\left(\frac{5}{3} \mathcal{W} u-\frac{1}{3} n u^{3}-\frac{\hbar^{2}}{8} n \partial_{x_{1}}^{2} u\right) & =-n u \partial_{x_{1}} V .
\end{aligned}
$$

Here we do not measure the error arising from the assumption that the stress tensor is scalar but we recover the classical hydrodynamic equations used in the literature at the semiclassical limit $(\hbar \rightarrow 0)$. 


\section{QUANTUM HYDRODYNAMIC AND QUANTUM ENERGY TRANSPORT MODELS}

4.3. Simplification of fluxes and QHD with slowly varying temperature. In three dimensions, the QHD model is more complicated and the relations of Lemma (4.1) are nonlocal and difficult to handle numerically. We would like, at least for the pressure tensor $\Pi$ and the energy flux $\Phi$, to give local expressions (as has been done for the isothermal Quantum Euler system [11]), i.e., to express $\nabla \cdot \Pi$ and $\nabla \cdot \Phi$ in a differential way involving only the extensive variables $n, n u, \mathcal{W}$, and the intensive ones $A, B, C$. Unfortunately we have not succeeded yet, but Theorem (4.2) constitutes one step in this direction. It gives us $\nabla \cdot \frac{\Pi}{C}$ and $\nabla \cdot \frac{\Phi}{C^{2}}$ instead of $\nabla \cdot \Pi$ and $\nabla \cdot \Phi$. If we suppose that the generalized temperature is slowly varying, we can suppose that

$$
\left|\frac{\nabla C}{C}\right|=\varepsilon \ll 1
$$

with $\varepsilon$ a small positive parameter.

Using Lemma (4.2), we obtain the following approximation for the divergence of the pressure tensor:

$$
\nabla \cdot \Pi=\nabla(n u \otimes B)+(\nabla B) \cdot n u-n \nabla\left(A C+\frac{B^{2}}{2}\right)+\mathcal{O}(\varepsilon) .
$$

Note that if we take $C=T$ to be a constant, we find the expression of $\nabla \cdot \Pi$ already found for the Isothermal Quantum Euler model [11].

In the same manner we obtain the following approximation for the energy flux:

$$
\nabla \cdot \Phi=(\Pi \nabla) \cdot B+\nabla \cdot(B \mathcal{W})-n u \cdot \nabla\left(A C+\frac{B^{2}}{2}\right)-\frac{\hbar^{2}}{8} \nabla \cdot(n \Delta B)+\mathcal{O}(\varepsilon) .
$$

In this last expression, we see that $\nabla \cdot \Phi$ depends on $\Pi$, and unfortunately we do not know any approximation for it and we have not been able to find one which is compatible with the approximation made for $\nabla \cdot \Pi$ in expression (4.44).

\section{Remarkable properties of QET}

5.1. Applications of the technical lemmas to QET. All the quantities expressed in the QET model can also be written with respect to the spectrum of the modified Hamiltonian $H(A, 0, C)$. Suppose $H(A, 0, C)$ has a discrete spectrum that we denote $\left(\lambda_{p}, \psi_{p}\right)_{p \in \mathbb{N}}$; then this spectrum is real and Lemma (3.2) allows us to compute the moments $n$ and $\mathcal{W}$ as has been done in Lemma (4.1). In the same lemma, we have given the expression for $\Pi$ and $\nabla \cdot \Pi$. In the next lemma, we complete this list for the QET model.

Lemma 5.1. Suppose $H(A, 0, C)$ has a discrete spectrum that we denote $\left(\lambda_{p}, \psi_{p}\right)_{p \in \mathbb{N}}$; then we have:

$$
\begin{gathered}
\mathbb{Q}=-\frac{\hbar^{4}}{16} \sum_{p \in \mathbb{N}}\left(s^{\prime}\right)^{-1}\left(-\lambda_{p}\right)\left(\nabla \psi_{p} \otimes \nabla \Delta \psi_{p}-2\left(\nabla \otimes \nabla \psi_{p}\right)^{2}+\nabla \Delta \psi_{p} \otimes \nabla \psi_{p}\right. \\
\left.-\Delta \psi_{p}\left(\nabla \otimes \nabla \psi_{p}\right)+2\left(\nabla \otimes \nabla \otimes \nabla \psi_{p}\right) \cdot \nabla \psi_{p}-\psi_{p}\left(\nabla \otimes \nabla \Delta \psi_{p}\right)\right) \\
\nabla \cdot \mathbb{Q}=-\frac{\hbar^{4}}{16} \sum_{p \in \mathbb{N}}\left(s^{\prime}\right)^{-1}\left(-\lambda_{p}\right)\left(-2\left(\nabla \otimes \nabla \psi_{p}\right) \cdot \nabla \Delta \psi_{p}+\Delta \Delta \psi_{p} \nabla \psi_{p}\right. \\
\left.+2\left(\nabla \otimes \nabla \Delta \psi_{p}\right) \cdot \nabla \psi_{p}-\psi_{p} \nabla \Delta \Delta \psi_{p}\right)
\end{gathered}
$$




$$
\begin{gathered}
\nabla \cdot \nabla \cdot \mathbb{Q}=-\frac{\hbar^{4}}{16} \sum_{p \in \mathbb{N}}\left(s^{\prime}\right)^{-1}\left(-\lambda_{p}\right)\left(\Delta \Delta \psi_{p} \Delta \psi_{p}+2 \nabla \psi_{p} \cdot \nabla \Delta \Delta \psi_{p}\right. \\
\left.-\psi_{p} \Delta \Delta \Delta \psi_{p}-2\left|\nabla \Delta \psi_{p}\right|^{2}\right) .
\end{gathered}
$$

We have also:

$$
\begin{aligned}
\nabla \cdot \nabla \cdot \Pi & =\frac{\hbar^{2}}{2} \sum_{p \in \mathbb{N}}\left(s^{\prime}\right)^{-1}\left(-\lambda_{p}\right)\left(\left|\Delta \psi_{p}\right|^{2}-\psi_{p} \Delta \Delta \psi_{p}\right), \\
\nabla \cdot(\mathcal{W} \mathrm{Id}) & =\frac{\hbar^{2}}{4} \sum_{p \in \mathbb{N}}\left(s^{\prime}\right)^{-1}\left(-\lambda_{p}\right)\left(2\left(\nabla \otimes \nabla \psi_{p}\right) \cdot \nabla \psi_{p}-\nabla \psi_{p} \Delta \psi_{p}-\psi_{p} \nabla \Delta \psi_{p}\right) .
\end{aligned}
$$

Proof. Let $\left\{e_{1}, e_{2}, e_{3}\right\}$ denote the standard basis of $\mathbb{R}^{3}$. We use Lemma (3.2) with:

- $\eta=(0,0,0)$ and $\alpha=e_{i}+e_{j}+2 e_{k}$ to get the coefficient $(i, j)$ of (5.1) after summation on $k$ and division by 2 ,

- $\eta=e_{j}$ and $\alpha=e_{i}+e_{j}+2 e_{k}$ to get the $i^{\text {th }}$ component of (5.2) after summation on $j$ and $k$ and division by 2 ,

- $\eta=e_{i}+e_{j}$ and $\alpha=e_{i}+e_{j}+2 e_{k}$ to get (5.3) after summation on $i, j$ and $k$ and division by 2 ,

- $\eta=e_{i}+e_{j}$ and $\alpha=e_{i}+e_{j}$ to get (5.4) after summation on $i$ and $j$,

- $\eta=e_{i}$ and $\alpha=2 e_{j}$ to get the $i^{\text {th }}$ component of (5.5) after summation on $j$ and division by 2 .

As has been done in Lemma (5.2), we now state some general properties involving $\Pi, \mathbb{Q}$ and some differential expressions of the extensive quantities $n, \mathcal{W}$ and intensive ones $A$ and $C$.

Theorem 5.2. Let $n, \mathcal{W}, \Pi, \mathbb{Q}$ and $A, C$ satisfy (2.33, 2.41-2.43). Then:

$$
\begin{aligned}
\nabla \cdot \frac{\Pi}{C}= & -n \nabla A-\mathcal{W} \nabla \frac{1}{C}+\frac{\hbar^{2}}{8} \Delta\left(n \nabla \frac{1}{C}\right) \\
\nabla \cdot \frac{\mathbb{Q}}{C^{2}}+\frac{1}{2 C} \operatorname{Tr} \mathbb{Q} \nabla \frac{1}{C}= & -(\Pi+\mathcal{W} I d) \cdot \frac{\nabla A}{C}+\frac{\hbar^{2}}{8 C}\left(n \nabla \Delta A+\mathcal{W} \nabla \Delta \frac{1}{C}+\nabla \cdot\left(\Pi \Delta \frac{1}{C}\right)\right. \\
& \left.+2 \nabla \cdot\left(\left(\nabla \otimes \nabla \frac{1}{C}\right) \Pi\right)+\Delta\left((\Pi+\mathcal{W} I d) \cdot \nabla \frac{1}{C}\right)\right) \\
& -\frac{\hbar^{4}}{64 C} \Delta\left(n \nabla \Delta \frac{1}{C}\right)
\end{aligned}
$$

Proof. For the first identity, we use (4.10) with $B=0$. For the second identity, we use again Lemma (3.4). Let $\mu(x)$ be a smooth test function; we compute:

$$
\left[W(\tilde{H}(\tilde{A}, 0, \tilde{C})), \mu|p|^{2} p\right]_{\hbar}=\left[\tilde{A}, \mu|p|^{2} p\right]_{\hbar}+\left[\tilde{C}|p|^{2}, \mu|p|^{2} p\right]_{\hbar}
$$


906 QUANTUM HYDRODYNAMIC AND QUANTUM ENERGY TRANSPORT MODELS

$$
\begin{aligned}
= & -i \hbar\left(-2 \mu(\nabla \tilde{A} \cdot p) p-\mu|p|^{2} \nabla \tilde{A}+\frac{\hbar^{2}}{4} \mu \nabla \Delta \tilde{A}\right. \\
& -2 \mu(\nabla \tilde{C} \cdot p)|p|^{2} p-\mu \nabla \tilde{C}|p|^{2}|p|^{2}+2 \tilde{C}(\nabla \mu \cdot p)|p|^{2} p \\
& -\frac{\hbar^{2}}{4}\left(-\mu \nabla \Delta \tilde{C}|p|^{2}+2(\nabla \mu \cdot p) \Delta \tilde{C} p+4((\nabla \otimes \nabla \tilde{C})(p \otimes p)) \cdot \nabla \mu\right. \\
& \left.\left.-2 \Delta \mu(\nabla \tilde{C} \cdot p) p-\Delta \mu \nabla \tilde{C}|p|^{2}\right)-\frac{\hbar^{4}}{16} \Delta \mu \nabla \Delta \tilde{C}\right) .
\end{aligned}
$$

Then due to the cyclicity of the trace, we write:

$$
\begin{array}{r}
\operatorname{Tr}\left(\left[\tilde{H}(\tilde{A}, \tilde{B}, \tilde{C}), W^{-1}\left(\mu|p|^{2} p\right)\right] \varrho_{n, \mathcal{W}}^{e q}\right)=0 \\
=\int\left[W(\tilde{H}(\tilde{A}, 0, \tilde{C})), \mu|p|^{2} p\right]_{\hbar} W\left(\varrho_{n, \mathcal{W}}^{e q}\right) \frac{d x d p}{(2 \pi \hbar)^{3}},
\end{array}
$$

which gives weakly the desired identity after integrations by parts and the change of variable given by $(2.15)$ (with $B=\tilde{B}=0$ ).

5.2. Simplification of fluxes and QET with slowly varying temperature.

As has been done for the QHD model, we can discuss the case of slowly varying temperature for the QET model. Let us denote again $\left|\frac{\nabla C}{C}\right|=\varepsilon \ll 1$. Using Theorem (5.2), we obtain the following approximation for the divergence of the pressure tensor:

$$
\nabla \cdot \Pi=-n \nabla(A C)+\mathcal{O}(\varepsilon) .
$$

Note that if we take $C=T$ to be a constant, we find the expression of $\nabla \cdot \Pi$ already found for the Quantum Drift-Diffusion model $[13,18,10]$.

For the divergence of the heat flux tensor, we find the following approximation:

$$
\nabla \cdot \mathbb{Q}=-(\Pi+\mathcal{W} \mathrm{Id}) \cdot \nabla(A C)+\frac{\hbar^{2}}{8} n \nabla \Delta(A C)+\mathcal{O}(\varepsilon),
$$

so that the currents can be approximated by

$$
\begin{gathered}
j_{n}=n \nabla(A C-V)+\mathcal{O}(\varepsilon) \\
j_{\mathcal{W}}=(\mathcal{W} \operatorname{Id}+\Pi) \cdot \nabla(A C-V)-\frac{\hbar^{2}}{8} n \nabla(\Delta(A C-V))+\mathcal{O}(\varepsilon) .
\end{gathered}
$$

Unfortunately again, we do not know any approximation for $\Pi$ and we have not been able to find one which is compatible with the approximation made for $\nabla \cdot \Pi$ in expression (5.9) - except in one dimension, where we see again two possibilities.

1. If we suppose that the transport is confined in dimension one (the momentum $p$ being confined on a line with coordinate $x_{1} \in \mathbb{R}$ ), and if we suppose that $\left|\frac{\partial_{x_{1}} C}{C}\right|=\varepsilon \ll 1$, the $1 \mathrm{D}$ QET model reads:

$$
\begin{array}{r}
\partial_{t} n+\partial_{x_{1}} j_{n}=0, \\
\partial_{t} \mathcal{W}+\partial_{x_{1}} j_{\mathcal{W}}+\left(\partial_{x_{1}} V\right) j_{n}=0,
\end{array}
$$

where

$$
\begin{aligned}
j_{n} & =-2 \partial_{x_{1}} \mathcal{W}-n \partial_{x_{1}} V \\
j_{\mathcal{W}} & =3 \mathcal{W} \partial_{x_{1}}(A C-V)-\frac{\hbar^{2}}{8} n \partial_{x_{1}}^{3}(A C-V)+\mathcal{O}(\varepsilon),
\end{aligned}
$$


with:

$$
\left(\begin{array}{c}
n \\
\mathcal{W}
\end{array}\right)=\int w_{n, \mathcal{W}}^{e q}\left(\begin{array}{c}
1 \\
\frac{p_{1}^{2}}{2}
\end{array}\right) \frac{d p_{1}}{2 \pi \hbar}
$$

The quantum local equilibrium is defined by

$$
w_{n, \mathcal{W}}^{e q}=W\left(\varrho_{n, \mathcal{W}}^{e q}\right)=W\left(\left(s^{\prime}\right)^{-1}(-H(A, 0, C))\right),
$$

where $H(A, 0, C)$ is the following modified Hamiltonian:

$$
\begin{aligned}
H(A, 0, C) & =W^{-1}\left(\frac{p_{1}^{2}}{2 C}+A\right) \\
& =-\hbar^{2}\left(\partial_{x_{1}}\left(\frac{1}{2 C} \partial_{x_{1}}\right)+\frac{1}{4} \partial_{x_{1}}^{2} \frac{1}{2 C}\right)+A .
\end{aligned}
$$

2. If the problem is in dimension 3 , but all the quantities depend only on one space variable ( $\operatorname{say} x_{1}$ ), then it is possible to show that the tensor $\Pi$ is diagonal (see Subsection 4.2.3). If we suppose that this tensor is scalar and equal to $2 \mathcal{W} / 3 \mathrm{Id}$, then the expressions of the currents (5.15) and (5.16) have to be replaced by:

$$
\begin{aligned}
j_{n} & =-\frac{2}{3} \partial_{x_{1}} \mathcal{W}-n \partial_{x_{1}} V \\
j_{\mathcal{W}} & =\frac{5}{3} \mathcal{W} \partial_{x_{1}}(A C-V)-\frac{\hbar^{2}}{8} n \partial_{x_{1}}^{3}(A C-V)+\mathcal{O}(\varepsilon) .
\end{aligned}
$$

\section{Conclusion and perspectives}

In this paper, we have given the expressions of the QHD and QET models with respect to the spectrum of the modified Hamiltonians $H(A, B, C)$ and $H(A, 0, C)$. We have then given some differential constraints that link the moments and their dual Lagrange multipliers. Concerning specifically the QHD model, a gauge invariance lemma allows us to simplify the model for irrotational flows. All these properties allow us to discuss some possible approximations of the models in the special cases of slowly varying temperature and/or of one dimensional flows which will facilitate future numerical approximations. The use of the commutator lemma should also allow us to study other quantum hydrodynamic-like models that have been derived with the same methodology based on the entropy principle, but involving other moments.

\section{REFERENCES}

[1] M.G. Ancona and G.J. Iafrate, Quantum correction of the equation of state of an electron gas in a semiconductor, Phys. Rev. B, 39, 9536-9540, 1989.

[2] M.G. Ancona and H.F. Tiersten, Macroscopic physics of the silicon inversion layer, Phys. Rev. B, 39, 7959-7965, 1987.

[3] A. Arnold, J.L. Lopez, P.A. Markowich and J. Soler, An analysis of quantum Fokker-Planck models: A Wigner function approach, Rev. Mat. Iberoamericana, 20, 771-814, 2004.

[4] A. Arnold and F. Nier, The two-dimensional Wigner-Poisson problem for an electron gas in the charge neutral case, Math. Methods Appl. Sci., 14(9), 595-613, 1991.

[5] P. Bhatnagar, E. Gross and M. Krook, A model for collision processes in gases. I. Small amplitude processes in charged and neutral one-component systems, Phys. Review, 94, 511-525, 1954.

[6] N. Ben Abdallah and A. Unterreiter, On the stationary quantum drift-diffusion model, Z. Angew. Math. Phys., 49(2), 251-275, 1998. 


\section{QUANTUM HYDRODYNAMIC AND QUANTUM ENERGY TRANSPORT MODELS}

[7] L.L. Chang, L. Esaki and R. Tsu, Resonant tunneling in semiconductor double barriers, Appl. Phys. Lett., 24, 593-595, 1974.

[8] R.C. Chen and J.L. Liu, A quantum corrected energy-transport model for nanoscale semiconductor devices, J. Comput. Phys., 204, 131-156, 2005.

[9] S. Datta, Nanoscale device modeling: the Green's function method, superlattices and microstructures, 28, 253-278, 2000.

[10] P. Degond, S. Gallego and F. Méhats, An entropic quantum drift-diffusion model for electron transport in resonant tunneling diodes, J. Comput. Phys., 221, 226-249, 2007.

[11] P. Degond, S. Gallego and F. Méhats, Isothermal quantum hydrodynamics: derivation, asymptotic analysis and simulation, SIAM Multiscale Model. Simul., 6(1), 246-272, 2007.

[12] P. Degond, S. Gallego, F. Méhats and C. Ringhofer, Quantum hydrodynamic and diffusion models derived from the entropy principle, Springer Lecture Notes in Mathematics, to be published.

[13] P. Degond, F. Méhats and C. Ringhofer, Quantum hydrodynamic models derived from the entropy principle, Contemp. Math., 371, 107-331, 2005.

[14] P. Degond, F. Méhats and C. Ringhofer, Quantum energy-transport and drift-diffusion models, J. Stat. Phys., 118(3-4), 625-665, 2005.

[15] P. Degond and C. Ringhofer, Quantum moment hydrodynamics and the entropy principle, J. Stat. Phys., 112(3-4), 587-628, 2003.

[16] W. Dreyer, M. Junk and M. Kunik, On the approximation of the Fokker-Planck equation by moment systems, Nonlinearity, 14, 881-906, 2001.

[17] F. Dubin, R. Melet, T. Barisien, R. Grousson, L. Legrand, M. Schott and V. Voliotis, Macroscopic coherence of a single exciton state in an organic quantum wire, Nature Phys., 2, 32-35, 2006.

[18] S. Gallego and F. Méhats, Entropic discretization of a quantum drift-diffusion model, SIAM J. Numer. Anal., 43(5), 1828-1849, 2005.

[19] C. Gardner, The quantum hydrodynamic model for semiconductor devices, SIAM J. Appl. Math., 54(2), 409-427, 1994.

[20] C. Gardner, Resonant tunneling in the quantum hydrodynamic model, VLSI Design, 3, 201-210, 1995.

[21] C. Gardner and C. Ringhofer, The smooth quantum potential for the hydrodynamic model, Phys. Rev. E, 53, 157-167, 1996.

[22] C. Gardner and C. Ringhofer, The Chapman-Enskog expansion and the quantum hydrodynamic model for semiconductor devices, VLSI Design, 10, 415-435, 2000.

[23] I. Gasser and A. Jügel, The quantum hydrodynamic model for semiconductors in thermal equilibrium, Z. Angew. Math. Phys., 48(1), 45-59, 1997.

[24] I. Gasser and P.A. Markowich, Quantum hydrodynamics, Wigner transforms and the classical limit, Asympt. Analysis, 14(2), 97-116, 1997.

[25] A. Jügel, Quasi-Hydrodynamic Semiconductor Equations, Progress in Nonlinear Differential Equations, Birkhäuser, 2001.

[26] A. Jügel and D. Matthes, A derivation of the isothermal quantum hydrodynamic equations using entropy minimization, Z. Angew. Math. Mech., 85, 806-814, 2005.

[27] A. Jügel, D. Matthes and J.P. Milisic, Derivation of new quantum hydrodynamic equations using entropy minimization, SIAM J. Appl. Math., 67, 46-68, 2006.

[28] A. Jüngel and R. Pinnau, A positivity preserving numerical scheme for a fourth-order parabolic equation, SIAM J. Num. Anal., 39(2), 385-406, 2001.

[29] C.D. Levermore, Moment closure hierarchies for kinetic theories, J. Stat. Phys., 83, 1021-1065, 1996.

[30] F. Nier, A stationary Schrödinger-Poisson system arising from the modeling of electronic devices, Forum Math., 2(5), 489-510, 1990.

[31] A. Unterreiter and R. Pinnau, The stationary current-voltage characteristics of the quantum drift-diffusion model, SIAM J. Num. Anal., 37(1), 211-245, 1999.

[32] E. Polizzi, Modélisation et simulations numériques du transport quantique balistique dans les nanostructures semi-conductrices, Ph.D. thesis, INSA, Toulouse, 2001.

[33] E. Polizzi and N. Ben Abdallah, Self-consistent three dimensional models for quantum ballistic transport in open systems, Phys. Rev. B, 66, 245-301, 2002.

[34] D. Robert, Autour de l'Approximation Semi-Classique, Birkhäuser, Boston, 1987. 